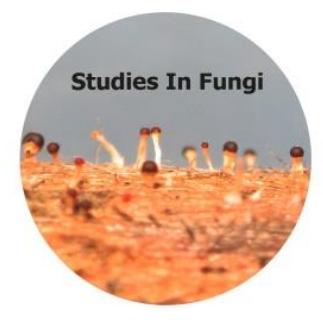

Studies in Fungi 3(1): 100-114 (2018) www.studiesinfungi.org ISSN 2465-4973 Article

Doi 10.5943/sif/3/1/12

Copyright $\odot$ Institute of Animal Science, Chinese Academy of Agricultural Sciences

\title{
New additions of macrolichens to the lichen flora of Arunachal Pradesh, India in Eastern Himalaya
}

\author{
Debnath $\mathbf{R}^{1}$, Khare $\mathbf{R}^{2}$, Gogoi $\mathbf{L}^{1}$, Upreti $\mathbf{D K}^{2}$ and Rout $\mathbf{J}^{1^{*}}$ \\ ${ }^{1}$ Department of Ecology and Environmental Science, Assam University, Silchar, Assam-788011, India \\ ${ }^{2}$ Lichenology Laboratory, National Botanical Research Institute, Rana Pratap Marg, Lucknow-226001, India
}

Debnath R, Khare R, Gogoi L, Upreti DK, Rout J 2018 - New additions of macrolichens to the lichen flora of Arunachal Pradesh, India in Eastern Himalaya. Studies in Fungi 3(1), 100-114, Doi $10.5943 / \mathrm{sif} / 3 / 1 / 12$

\begin{abstract}
Lichen exploration of West Kameng district and Tawang district of Arunachal Pradesh state, India located in the Eastern Himalayas reveal seventy nine lichens represented primarily by foliose (73\%), a few fruticose (15\%), dimorphic (8\%), leprose $(2 \%)$ and crustose $(1 \%)$ types. Of these, seventeen species, one fruticose, sixteen foliose growth forms, belonging to 12 genera and 5 families are recorded as new additions to the lichen flora of Arunachal Pradesh state. The lichens were sampled at an elevation ranging from 1618 meter to 4509 meter. The diagnostic features of new additions to macrolichens of Arunachal Pradesh based on the phytogeographic affinities and distribution of species is briefly described.
\end{abstract}

Keywords - Foliose - Fruticose - Elevation - Distribution

\section{Introduction}

Lichens are the symbiotic association between a fungus (mycobiont) and an alga (phycobiont) or cyanobacteria (cyanobiont). They comprise a unique group which are among the most significant indicators of environment and also sensitive towards habitat variation (Wolseley et al. 1994, Rai et al. 2011). India is blessed with rich lichen biodiversity contributing $15 \%$ of total global lichen flora (Singh \& Sinha 1997, Upreti 1998). Most of the parts of the country are still unexplored and lichenologists are trying to explore new records of lichens from India. Recently Polymeridium cinereonigricans (Vain.) R.C. Harris, P. pleurothecium R.C. Harris and P. submuriforme Aptroot are recorded as new to India (Ingle et al. 2017). A total of 49 species belongs to 26 genera and 14 families of lichens species are recorded recently from the mangrove forests of Bhitarkanika Wildlife Sanctuary and National Park (Odisha) for the first time (Panda et al. 2017). Lichens along with cyanobacteria contribute a significant role for forest nitrogen fixation and also have various industrial applications. Lichens are being used in several ways by different communities of ethnic people. The ethnic use of seven species of lichens belonging to four families is recently recorded from Himalayan country Nepal (Devkota et al. 2017). Secondary metabolites produced by such organisms are of great relevance to medical science (Rout et al. 2005). Lichens are also used for pollution monitoring (Rout 2007) and in dating rocks. With realization of potential impacts of global climate shifts, the lichen flora of polar and alpine regions have assumed greater significance. The vegetation of Arunachal Pradesh has been categorized into six broad types, viz.: tropical, 
subtropical, temperate, sub-alpine, alpine and secondary forests (Kaul \& Haridasan 1987). Research related to lichen diversity in North-Eastern region of the country is only beginning to get momentum in the last couple of decades or so (Singh \& Pinokiyo 2004, Pinokiyo et al. 2008, Rout et al. 2005, Rout 2007, Rout et al. 2010, Rout et al. 2013, Sinha \& Jagadeesh 2011, Daimari et al. 2014, Upreti et al. 2015, Dey et al. 2015, Devi et al. 2015, Logesh et al. 2017). Arunachal Pradesh, a state in the North East India is one of the most high lichen diversity region of India. Much of the area in the state, including the Himalayan foothills and the Patkai hills, are home to Eastern Himalayan broadleaf forests (Upreti 1997). Lichen survey in Arunachal Pradesh was initiated by Awasthi (1961). Other lichenologist such as Upreti 1985a, Upreti 1985b, Rout et al. 2004a, Rout et al. 2004b, Dubey \& Rout 2007, Dubey 2009, Bajpai et al. 2016, Singh et al. 2018, Sinha et al. 2018 including Awasthi 1982, have also made important contributions to the study of lichens of Arunachal Pradesh. However several areas in the state still remained unexplored. Hence, an extensive field exploration was made covering West Kameng and Tawang districts of the state of Arunachal Pradesh leading to some new additions to the lichen flora of the state.

\section{Materials \& Methods}

The study has been carried out in West Kameng and Tawang districts of western part of the state of Arunachal Pradesh (Fig. 1). The West Kameng district (area 7,422 km²) lies between $26^{\circ} 54^{\prime}$ to $28^{\circ} 01^{\prime} \mathrm{N}$ and $91^{\circ} 30^{\prime}$ to $92^{\circ} 40^{\prime} \mathrm{E}$. The district is bordered by Tibet region in the north, Tawang and East Kameng districts in the northwest and east, respectively. The southern boundary adjoins Sonitpur and Darrang districts of the state of Assam and Bhutan as international border in the West. The district is mostly mountainous. The vegetation types range from tropical to alpine between altitudinal ranges of 500 meter to 6000 meter. The Tawang district (area 2,172 $\mathrm{km}^{2}$ ) lies between $27^{\circ} 25^{\prime}$ to $27^{\circ} 52^{\prime} \mathrm{N}$ and $91^{\circ} 16^{\prime}$ to $91^{\circ} 59^{\prime} \mathrm{E}$. It is surrounded by Tibet in the north, Bhutan in the south-west and Sela ranges separate it from West Kameng district in the east. The vegetation type ranges from temperate to alpine covering altitude of 1500 meter to 6000 meter. Five study sites in each district, viz. Dirang, Sangti, Bartse, Tsangeso, Sela in West Kameng district and another five viz., Negroteng, Pangang Teng Tso(PTTSO), Bangajang, Nagula, Bumla in Tawang district were selected (Table 1). The study sites were chosen so as to represent ten progressively different elevations varying from Dirang to Bumla.

The study sites were stratified into three different vegetation with regard to the elevation such as Oak-Rhododendron mixed vegetation (1600-2500 meter), Rhododendron-rich middle subalpine forest (2500-3500 meter) and higher altitude alpine grassland with abies vegetation (3500-4500 meter). The specimens were collected from different substrata such as soil, rock, shrubs, tree trunks and twigs from different localities of West Kameng and Tawang districts of the state during September 2012 and November 2013. The collected specimens were properly dried, labeled and investigated morphologically, anatomically and chemically. Standard keys of Awasthi 2007, Divakar \& Upreti 2005 were used for the identification. The morphology was studied using a Labo Med Digi Zoom dissecting microscope, and the anatomical details were studied using a Leica TM DM 500 optical microscope. The color spot tests (K, C, KC, P test) and Thin Layer Chromatography (TLC) (Culberson \& Kristiansen 1970, Culberson 1972, Santesson 1973, Awasthi 1988, 1991) were performed. The chromatogram was developed using the solvent system A (tolune: dioxane: acetic acid::180: 60: $80 \mathrm{ml}$ ). Identified samples were deposited in the Department of Ecology and Environmental Science, Assam University, Silchar, Assam (AUS). A voucher specimen of each taxa has also been submitted to the herbarium of National Botanical Research Institute (NBRI), Lucknow (LWG), India. Few species were categorized as new addition to lichen flora of Arunachal Pradesh based on the annotated checklist (Singh \& Sinha 2010) and some of the recent reports on Indian lichens (Bajpai et al. 2016, Singh et al. 2018, Sinha et al. 2018).

\section{Results}

A total of 79 species belonging to 32 genera and 18 families of lichen have been identified from West Kameng and Tawang District of Arunachal Pradesh (Table 2). Three species belonging 
to 2 genera and 1 family of fruticose lichens and five species belonging to 4 genera and 3 families of foliose lichens are identified common from both the districts. Seventeen species belonging to twelve genera and five families were identified as new additions to the lichen flora of Arunachal Pradesh. The Parmeliaceae family was the most dominant in the area. Both the districts exhibited luxuriant growth of foliose lichens with 54 species of this type. Nagula was found to be the most lichen rich area during the course of the present study (Fig.4).

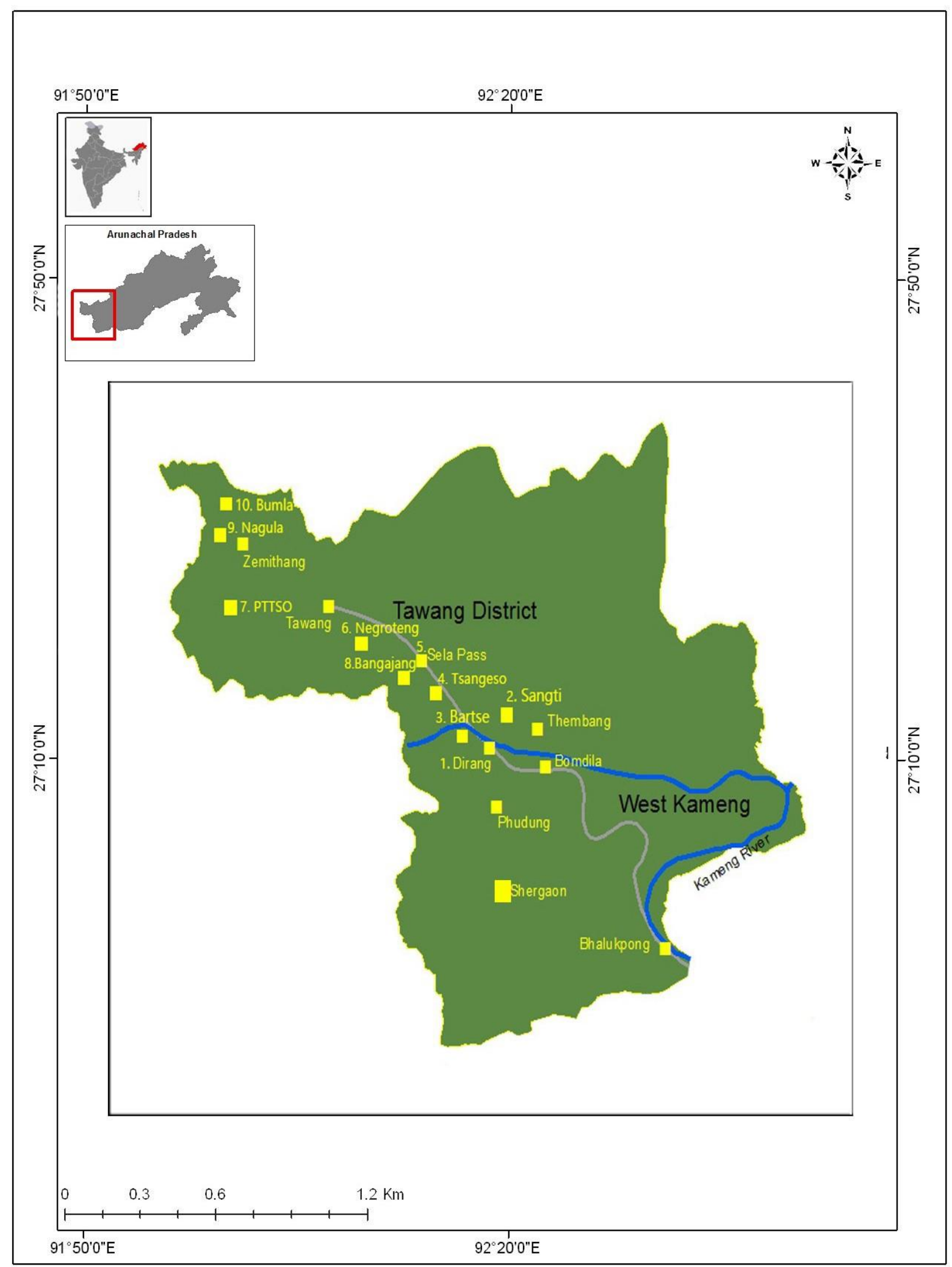

Fig. 1 - Map showing sites (1-10) of lichen collection in West Kameng and Tawang district 
Table 1 Lichen collection sites and their elevation.

\begin{tabular}{llllll}
\hline District & $\begin{array}{l}\text { Collection } \\
\text { sites }\end{array}$ & $\begin{array}{l}\text { Latitude } \\
(\mathbf{N})\end{array}$ & $\begin{array}{l}\text { Longitude } \\
(\mathbf{E})\end{array}$ & $\begin{array}{l}\text { Elevation } \\
(\mathbf{m})\end{array}$ & $\begin{array}{l}\text { Temp } \\
\left({ }^{\circ} \mathbf{C}\right)\end{array}$ \\
\hline West Kameng & & & & \\
& 1. Dirang & $27^{\circ} 25^{\prime} 24.0^{\prime \prime}$ & $92^{\circ} 18^{\prime} 10.8^{\prime \prime}$ & 1602 & 20 \\
& 2.Sangti & $27^{\circ} 26^{\prime} 01.2^{\prime \prime}$ & $92^{\circ} 07^{\prime} 12.2^{\prime \prime}$ & 2330 & 18 \\
& 3.Bartse & $27^{\circ} 17^{\prime} 57.8^{\prime \prime}$ & $92^{\circ} 15^{\prime} 32.6^{\prime \prime}$ & 2727 & 17 \\
& 4.Tsangeso & $27^{\circ} 43^{\prime} 14.1^{\prime \prime}$ & $91^{\circ} 49^{\prime} 18.5^{\prime \prime}$ & 3715 & 18 \\
& 5.Sela & $27^{\circ} 29^{\prime} 0.7^{\prime \prime}$ & $92^{\circ} 06^{\prime} 39.6^{\prime \prime}$ & 3837 & 18 \\
\hline Tawang & & & & \\
& & & & \\
& 6.Negroteng & $27^{\circ} 30^{\prime} 30.0^{\prime \prime}$ & $92^{\circ} 00^{\prime} 05.2^{\prime \prime}$ & 3802 & 20 \\
& 7.PTTSO & $27^{\circ} 38^{\prime} 17.2^{\prime \prime}$ & $91^{\circ} 51^{\prime \prime} 25.2^{\prime \prime}$ & 3916 & 14 \\
& 8.Bangajang & $27^{\circ} 29^{\prime} 54.5^{\prime \prime}$ & $92^{\circ} 01^{\prime \prime} 17.2^{\prime \prime}$ & 4146 & 8 \\
& 9.Nagula & $27^{\circ} 41^{\prime} 33.3^{\prime \prime}$ & $91^{\circ} 51^{\prime} 07.5^{\prime \prime}$ & 4290 & 10 \\
& 10.Bumla & $27^{\circ} 43^{\prime \prime} 14.3^{\prime \prime}$ & $91^{\circ} 53^{\prime} 31.2^{\prime \prime}$ & 4509 & 10 \\
\hline
\end{tabular}

\section{Taxonomy}

Bryoria tenuis (Dahl) Brodo \& D. Hawksw. (Family: Parmeliaceae)

Fig. 2.1

Thallus erect to decumbent, to $8 \mathrm{~cm}$ long, often dying at base; dark brown to black in basal region, paler brown towards apices, branched; main branch to $0.5 \mathrm{~mm}$ in diam; lateral spinules present; pseudocyphellae fissural, usually dark; isidia, soredia and isidioid spinules absent. Apothecia absent in Indian specimens.

Basionym - Alectoria tenuis Dahl, Meddl. Groenl. 150(2): 144.1950. Type: Greenland, Julianehaab District. Igalikfjord. Eqaluit, 9 Aug. 1937. E. Dahl (O-holotype)

Known distribution - distributed in the temperate regions of India (Uttaranchal and West Bengal-Darjeeling District), in boreal and arctic regions of Europe and North America.

Material examined - India, Arunachal Pradesh, Tawang, PTTSO, substratum-tree twig; 24 Sept. 2012; R. Debnath; AUS/JR-RD/APPT/12-11, 12-017720(LWG); Negroteng; 23 Sept. 2012; R. Debnath; AUS/JR-RD/APN/12-06, 12-017723(LWG); LWG Accession no: 29490, 29489

Cetrelia collata (Nyl.) W.Culb. \& C.Culb. (Family: Parmeliaceae)

Fig. 2.2

Thallus to $15 \mathrm{~cm}$ across; lobes to $25 \mathrm{~mm}$ wide; upper side pale brownish, with wider than 1 mm diam. pseudocyphellae; lacking isidia and soredia; lower side black, punctuate or not, with few rhizines.

Basionym - Platysma collatum Nylander, Flora 70: 134. 1887. Type: China, Yunnan, Delavay 1590(H-NYL 36116-holotype).

$\equiv$ Cetraria collate (Nyl.) Muller Argoviensis, Nuov. Giorn. Bot. Ital. 24: 192.1892.

Known distribution - reported from temperate regions of India (Sikkim), Nepal, China.

Material examined - India, Arunachal Pradesh, West Kameng, Sela; substratum-tree bark; 23 Sept. 2012; R. Debnath; AUS/JR-RD/APSE/12-11; 12-017727(LWG).

Cetrelia olivetorum (Nyl.)W. Culb. \& C.Culb. (Family: Parmeliaceae)

Fig. 2.3

Thallus to $14 \mathrm{~cm}$ across; lobes to $18 \mathrm{~mm}$ wide; upper side tan to brownish with small, upto $0.5 \mathrm{~mm}$ wide pseudocyphellae; lobe margins with farinose; lower side black, rarely punctate, with few rhizines.

Basionym - Parmelia olivetorum Nylander, Nat. Sallsk.Fauna Fl. Fenn. Forhandl. N.S. 5: 180. 1866. Type: Switzerland (H-ACH-1327-lectotype).

Known distribution - widespread in temperate regions of India (Uttaranchal), Nepal, China, Japan, Europe and North America.

Material examined - India, Arunachal Pradesh, West Kameng, Bartse; substratum: tree bark; 22 Sept. 2012; R. Debnath; AUS/JR-RD/APB/12-07, 12-017797(LWG). 
Thallus usually monophyllus, to $12 \mathrm{~cm}$ across, umbilicate, rather thick, leathery; upper side light brownish to brownish red, white to dark pruinose; lower side black, with dense, thick, stumpy, coralloid rhizinomorphs. Perithecia pale red; ascospores ellipsoid, 9-12 x (5-) 6-9 $\mu \mathrm{m}$.

三Dermatocarpon rhizinosum Choisy,Bull. Soc. Bot. France 78: 455.1931. non D. rhizinosum (Mull. Arg.) Zahlbr.

Known distribution - widespread in subtropical to lower temperate regions of India (Himachal Pradesh, Jammu \& Kashmir, Maharastra, Rajasthan-Mt. Abu. Tamil Nadu-Nilgiri \& Palni Hills, Uttaranchal), Nepal, China and Eastern Europe.

Material examined - India, Arunachal Pradesh, Tawang, Bumla; substratum-rock; 26 Sept. 2012; R. Debnath; AUS/JR-RD/APUL1/12-01(AUS), 12-017754(LWG); adjacent areas of Nagula lake; substratum-rock; 12 Nov. 2013; R. Debnath; AUS/JR-RD/AP/13-55.

Everniastrum neocirrhatum (Hale \& Wirth) Hale. (Family: Parmeliaceae)

Fig. 2.5

Thallus to $19 \mathrm{~cm}$ across; lobes $0.5-1.5(-2.5) \mathrm{mm}$ wide; upper side grey, lacking isidia and soredia; lower side brown black, with short scattered rhizines. Apothecia to $10 \mathrm{~mm}$ in diam.; ascospores(14-)16-22 x (6-) 8-10 $\mu \mathrm{m}$.

Basionym - Parmelia neocirrhata Hale \& Wirth, Phytologia 22: 37.1971.

$\equiv$ Cetrariastrum neocirrhatum (Hale \& Wirth) W. Culberson \& C. Culberson. Bryologist 84: 289.1981.p.288.fig.10.

Known distribution - distributed in India (Nagaland), Mexico.

Material examined - India, Arunachal Pradesh, Tawang, adjacent areas of Nagula Lake; substratum-tree bark; 25 Sept. 2012; R. Debnath; AUS/JR-RD/APNL/12-47, 12-017783(LWG); West Kameng, Dwangbu, Sangti; substratum-tree bark; 9 Nov. 2013; R. Debnath; AUS/JRRD/AP/13-20, AUS/JR-RD/AP/13-68.

Hypogymnia alpina D.D.Awasthi. (Family: Parmeliaceae)

Fig. 2.6

Thallus adnate, branched; lobes to $1.5 \mathrm{~mm}$ wide, apically somewhat swollen; upper side greybrown to blackish, lacking soredia and isidia. Apothecia goblet shaped, $2 \mathrm{~mm}$ in diam; ascospores 5-10 x 4-6 $\mu \mathrm{m}$.

Known distribution - reported from India (Sikkim, Uttarakhand).

Material examined - India, Arunachal Pradesh, Tawang, Bumla; substratum- tree bark; 26 Sept. 2012; R. Debnath; AUS/JR-RD/APTL/12-02, 12-017777(LWG); Tawang, Bumla; substratum-tree bark; 13 Nov. 2013; AUS/JR-RD/AP/13-32, 15-025646(LWG).

Hypogymnia enteromorpha (Ach.) Nyl. Nylander. (Family: Parmeliaceae)

Fig. 2.7

Thallus branched, lobes compact to discrete, lacking adventitive branchlets; upper side greywhite to yellowish grey; isidia and soredia absent; Apothecia pedicellate and to $20 \mathrm{~mm}$ in diam; ascospores 6.5-8.3 x 3.8-5 $\mu \mathrm{m}$.

Basionym - Parmelia enteromorpha Acharius.Meth. Lich.: 252.1803; Bitter 1901: 233, tab. 11. Fig. 11.

Known distribution - reported from alpine regions of India (Sikkim), Japan, Korea, Tasmania and North America.

Material examined - India, Arunachal Pradesh, Tawang, adjacent areas of Nagula lake; substratum-tree bark; 25 Sept. 2012; R. Debnath; AUS/JR-RD/APNL/12-42, 12-017759(LWG)

Hypogymnia pseudohypotrypa(Asahina)A.Singh. (Family: Parmeliaceae)

Fig. 2.8

Thallus branched; lobes to $6 \mathrm{~mm}$ wide; upper side yellowish grey to grey-brown; isidia and soredia absent; Apothecia pedicellate, to $15 \mathrm{~mm}$ in diam.

Basionym - Parmelia pseudohypotrypa Asahina in Nuno, J. Jap.Bot. 39: 89.1964.

Known distribution-reported from upper temperate regions of India (Sikkim, West-BengalDarjeeling District), China and Tibet. 
Table 2 Species composition of lichens from West Kameng and Tawang district of Arunachal Pradesh during the study period.

\begin{tabular}{|c|c|c|c|c|c|c|c|c|c|c|c|c|c|}
\hline & Species & Sub & GF & DI & $\mathbf{S A}$ & BS & TS & $\mathbf{N E}$ & SE & PT & BA & NA & BU \\
\hline 1 & Acroscyphus sphaerophoroides Lev. & $\mathrm{T}$ & Fr. & & & & & & & & & + & \\
\hline 2 & Bryoria asiatica (Du Rietz) Brodo \& D. Hawksw. & $\mathrm{C}$ & Fr. & & + & & & & & & & & \\
\hline 3 & Bryoria confusa (D.D.Awasthi) Brodo \& D.Hawksw. & $\mathrm{C}$ & Fr. & & & & & & & & & & + \\
\hline 4 & Bryoria himalayana(Mot.) Brodo \& D.Hawksw. & $\mathrm{C}$ & Fr. & & & & & & & & + & & \\
\hline 5 & Bryoria tenuis(Dahl) Brodo \& D.Hawksw.* & $\mathrm{C}$ & Fr. & & & & & + & & + & & & \\
\hline 6 & Candelaria sp. & $\mathrm{T}$ & $\mathrm{F}$ & & & & & & & & & + & \\
\hline 7 & Canomaculina subsumpta(Nyl.) Elix & $\mathrm{C}$ & $\mathrm{F}$ & & & & & & & & & & + \\
\hline 8 & Cetrelia cetrarioides(Del. ex Duby) W. Culb. \& C.Culb. & $\mathrm{C}$ & $\mathrm{F}$ & & & & + & & & & & & \\
\hline 9 & Cetrelia collata(Nyl.) W. Culb. \& C. Culb.* & $\mathrm{C}$ & $\mathrm{F}$ & & & & & & + & & & & \\
\hline 10 & Cetrelia braunsiana(Mull. Arg.) W. Culb. \& C.Culb. & $\mathrm{C}$ & $\mathrm{F}$ & & & + & & & & & & & \\
\hline 11 & Cetrelia olivetorum(Nyl.) W. Culb. \& C. Culb.* & $\mathrm{C}$ & $\mathrm{F}$ & & & + & & & & & & & \\
\hline 12 & Cladonia coccifera $(\mathrm{L}$.$) Willd.$ & $\mathrm{T}$ & $\mathrm{D}$ & & & & & & & + & & & \\
\hline 13 & Cladonia pyxidata(L.) Hoffim. & $\mathrm{T}$ & $\mathrm{D}$ & & & & & & & & & + & \\
\hline 14 & Cladonia rangiferina $(\mathrm{L}$.$) Waber ex F.H. Wigg.$ & $\mathrm{T}$ & Fr. & & & & & & & & & + & \\
\hline 15 & Cladonia stellaris (Opiz) Pouzar \& Vezda & $\mathrm{T}$ & Fr. & & & & & & & & & + & \\
\hline 16 & $\begin{array}{l}\text { Cladonia yunnana(Vain) Abbayes ex J.C. Wei \& Y.M. } \\
\text { Jiang }\end{array}$ & $\mathrm{T}$ & $\mathrm{D}$ & & & & & & & & & + & \\
\hline 17 & Coccocarpia erythroxyli(Spreng.) Swinsc. \& Krog & $\mathrm{T}$ & $\mathrm{F}$ & + & & + & & & & & & & \\
\hline 18 & $\begin{array}{l}\text { Coccocarpia palmicola(Spreng.) Arvidss. \& D.J. } \\
\text { Galloway }\end{array}$ & $\mathrm{C}$ & $\mathrm{F}$ & + & & & & & & & & & \\
\hline 19 & Crysothrix candelaris(L.) J.R. Laundon & S & $\mathrm{L}$ & & & & & & & & & + & \\
\hline 20 & Dermatocarpon vellereum Zschacke* & $S$ & $\mathrm{~F}$ & & & & & & & & & & + \\
\hline 21 & Everniastrum cirrhatum (Fr.) Hale & $\mathrm{C}$ & $\mathrm{F}$ & + & + & & + & & + & + & & & \\
\hline 22 & Everniastrum neocirrhatum (Hale \& Wirth) Hale* & $\mathrm{C}$ & $\mathrm{F}$ & & & & & & & & & + & \\
\hline 23 & Everniastrum nepalense(Taylor) Hale & $\mathrm{C}$ & $\mathrm{F}$ & & & & & & + & & + & & \\
\hline 24 & Flavopunctelia flaventior(Stirton) Hale & $\mathrm{C}$ & $\mathrm{F}$ & & & & + & & & & & & \\
\hline 25 & Heterodermia boryi $(\mathrm{Fee})$ Kr.P. Singh \& S.R.Singh & $\mathrm{C}$ & $\mathrm{F}$ & & & & + & + & & & & & \\
\hline 26 & Heterodermia diademata (Taylor) D.D.Awasthi & $\mathrm{B}$ & $\mathrm{F}$ & & + & & & & & & & & \\
\hline 27 & Heterodermia leucomelos(L.) Poelt & $\mathrm{B}$ & $\mathrm{F}$ & & & + & & & & & & & \\
\hline 28 & Heterodermia pseudospeciosa (Kurok.) W. Culb. & $\mathrm{B}$ & $\mathrm{F}$ & + & & + & & & & & & & \\
\hline 29 & Heterodermia speciosa (Wulf.) Trevis. & $\mathrm{B}$ & $\mathrm{F}$ & + & & & & & & & & & \\
\hline
\end{tabular}


Table 2 Continued.

\begin{tabular}{|c|c|c|c|c|c|c|c|c|c|c|c|c|c|}
\hline & Species & Sub & GF & DI & $\mathbf{S A}$ & BS & TS & $\mathbf{N E}$ & SE & PT & $\mathbf{B A}$ & NA & $\mathbf{B U}$ \\
\hline 30 & Hypogymnia alpina D.D. Awasthi* & B & $\mathrm{F}$ & & & & & & & & & & + \\
\hline 31 & Hypogymnia enteromorpha (Ach.) Nyl.* & $\mathrm{B}$ & $\mathrm{F}$ & & & & & & & & & + & \\
\hline 32 & Hypogymnia physodes (L.) Nyl. & B & $\mathrm{F}$ & & & & & + & & & & & \\
\hline 33 & $\begin{array}{l}\text { Hypogymnia } \quad \text { pseudobitteriana } \\
\text { D.D.Awasthi }\end{array}$ & $\mathrm{B}$ & $\mathrm{F}$ & & & & & + & & & & & \\
\hline 34 & Hypogymnia pseudohypotrypa (Asahina) A.Singh* & $\mathrm{B}$ & $\mathrm{F}$ & & + & & & & & & & & \\
\hline 35 & Hypogymnia thamsoniana (Mull. Arg.) D.D. Awasthi* & $\mathrm{B}$ & $\mathrm{F}$ & & + & & & & & & & & \\
\hline 36 & Hypogymnia vittata (Ach.) Gasil. & $\mathrm{C}$ & $\mathrm{F}$ & & + & & + & & & + & & & \\
\hline 37 & Hypotrachyna infirma (Kurok.) Hale & $\mathrm{C}$ & $\mathrm{F}$ & & & + & & & & & & & \\
\hline 38 & Leptogium cyanescens (Rabenh.) Korb. & $\mathrm{C}$ & $\mathrm{F}$ & + & & & & & & & & & \\
\hline 39 & Leptogium denticulatum $\mathrm{Nyl}$. & $\mathrm{C}$ & $\mathrm{F}$ & & + & & & & & & & & \\
\hline 40 & Leptogium trichophorum Mull. Arg. & $\mathrm{C}$ & $\mathrm{F}$ & & & & & & + & & & & \\
\hline 41 & Lepraria sp. & $\mathrm{T}$ & $\mathrm{L}$ & & & & & & & & & + & \\
\hline 42 & Lethariella cladonioides (Nyl.) Krog & $\mathrm{T}$ & Fr. & & & & & & & & + & & \\
\hline 43 & Lobaria retigera (Bory) Trev. & $\mathrm{C}$ & $\mathrm{F}$ & + & + & & & & & & & + & \\
\hline 44 & Melanelia stygia (L.) Essl. & $\mathrm{S}$ & $\mathrm{F}$ & & & & & & & & & + & \\
\hline 45 & Myelochroa metarevoluta (Asahina) Elix \& Hale* & $\mathrm{S}$ & $\mathrm{F}$ & & & & & & & & & + & \\
\hline 46 & Nephromopsis nephromoides (Nyl.) Ahti \& Randl. & $\mathrm{C}$ & $\mathrm{F}$ & & & + & & & & & & & \\
\hline 47 & Nephromopsis pallescens (Schaer.) Park & $\mathrm{C}$ & $\mathrm{F}$ & & & + & & & & & & & \\
\hline 48 & $\begin{array}{l}\text { Parmelinella chozoubae (Kr. P.Singh \& Sinha) Elix \& } \\
\text { Pooprang* }\end{array}$ & $\mathrm{S}$ & $\mathrm{F}$ & + & & & & & & & & & \\
\hline 49 & Parmelaria subthomsonii D.D.Awasthi & $\mathrm{C}$ & $\mathrm{F}$ & + & & & & & & & & & \\
\hline 50 & Parmotrema praesorediosum (Nyl.) Hale* & $\mathrm{C}$ & $\mathrm{F}$ & & & & & & & + & & & \\
\hline 51 & Parmotrema reticulatum(Taylor) Choisy & $\mathrm{C}$ & $\mathrm{F}$ & + & & + & & & & & & & \\
\hline 52 & Parmotrema saccatilobum(Taylor) Hale* & $\mathrm{C}$ & $\mathrm{F}$ & & & & & & & & & + & \\
\hline 53 & Parmotrema tinctorum( Despr. ex Nyl.) Hale & $\mathrm{C}$ & $\mathrm{F}$ & & & + & & & & & & & \\
\hline 54 & Peltigera collina(Ach.) Schrad.* & $\mathrm{T}$ & $\mathrm{F}$ & & & & & & & & + & & \\
\hline 55 & Peltigera polydactylon(Neck.) Hoffm. & $\mathrm{T}$ & $\mathrm{F}$ & + & & & & & & & & & \\
\hline 56 & Peltigera rufescens(Weiss) Humb. & $\mathrm{T}$ & $\mathrm{F}$ & & & & & & & & + & & \\
\hline 57 & Physcia caesia(Hoffm.) Furnr.* & $\mathrm{C}$ & $\mathrm{F}$ & & & & & & & & & + & \\
\hline 58 & Ramalina hossei Vain. & $\mathrm{C}$ & Fr. & + & & & & & & & & & \\
\hline
\end{tabular}


Table 2 Continued.

\begin{tabular}{|c|c|c|c|c|c|c|c|c|c|c|c|c|c|}
\hline & Species & Sub & GF & DI & $\mathbf{S A}$ & BS & TS & $\mathbf{N E}$ & SE & PT & $\mathbf{B A}$ & $\mathbf{N A}$ & BU \\
\hline 59 & Ramalina sinensis Jatta & $\mathrm{C}$ & Fr. & + & & & & & & & & & \\
\hline 60 & Rhizocarpon geographicum(L.) DC. & $\mathrm{S}$ & Cr. & & & & & & & & & + & \\
\hline 61 & Stereocaulon alpinum Laurer & $\mathrm{T}$ & $\mathrm{D}$ & & & & & & & & + & & \\
\hline 62 & $\begin{array}{l}\text { Stereocaulon foliolosum Nyl. var. botryophorum (Mull. } \\
\text { Arg.) I.M. Lamb }\end{array}$ & $\mathrm{T}$ & $\mathrm{D}$ & & & & & & & & & + & \\
\hline 63 & Stereocaulon himalayense D.D.Awasthi \& I.M.Lamb & $\mathrm{T}$ & $\mathrm{D}$ & & & & & & & & & + & + \\
\hline 64 & Sticta nylanderiana Zahlbr.* & $\mathrm{C}$ & $\mathrm{F}$ & & + & & & & & & & & \\
\hline 65 & Sticta weigelii(Ach.)Vain. & $\mathrm{C}$ & $\mathrm{F}$ & + & & & & & & & & & \\
\hline 66 & Sulcaria sulcata(Lev.) Bystr. ex Brodo \& D.Hawksw. & $\mathrm{C}$ & Fr. & + & & & & & & & & & \\
\hline 67 & Thamnolia vermicularis(Sw.) Schaer. var. vermicularis & $\mathrm{T}$ & Fr. & & & & & & & & & + & \\
\hline 68 & Umbilicaria indica Frey var. indica & $\mathrm{S}$ & $\mathrm{F}$ & & & & & & & & + & + & + \\
\hline 69 & Umibilicaria vellea (L.) Ach. em. Frey* & $S$ & $\mathrm{~F}$ & & & & & & & & & + & \\
\hline 70 & Usnea aciculifera Vain. & $\mathrm{C}$ & $\mathrm{F}$ & + & & & & & + & & & & \\
\hline 71 & Usnea bismolliuscula Zahlbr. & $\mathrm{C}$ & $\mathrm{F}$ & & + & & & & & & & & \\
\hline 72 & Usnea cineraria Mot. & $\mathrm{C}$ & $\mathrm{F}$ & & + & & & & & & & & \\
\hline 73 & Usnea dendritica Stirt. & $\mathrm{C}$ & $\mathrm{F}$ & & + & & & & & & & & \\
\hline 74 & Usnea himantodes Stirt. & $\mathrm{C}$ & $\mathrm{F}$ & & & & & & & & + & & \\
\hline 75 & Usnea longissima Ach. & $\mathrm{C}$ & $\mathrm{F}$ & & + & & + & + & & & & & \\
\hline 76 & Usnea misamisensis(Vain.) Mot. & $\mathrm{C}$ & $\mathrm{F}$ & & & & + & & & & & & \\
\hline 77 & Usnea orientalis Mot. & $\mathrm{C}$ & $\mathrm{F}$ & & & & & & & + & + & & \\
\hline 78 & Usnea thamsonii Stirt. & $\mathrm{C}$ & $\mathrm{F}$ & & + & & + & & & + & & & \\
\hline 79 & Usnea fragilis Stirt. & $\mathrm{C}$ & $\mathrm{F}$ & & & & & & & & + & & \\
\hline
\end{tabular}

Note: Sub-Substratum, GF-Growth Form, C-Corticolous, T-Terricolous, S-Saxicolous, F-Foliose, Fr.-Fruticose, Cr.- Crustose D-Dimorphic, LLeprose; BA-Bangajang, BS-Bartse, BU-Bumla, DI-Dirang, NA-Nagula Lake, NE-Negroteng, PT-PTTSO, TS-Tsangeso, SA-Sangti \& SE-Sela.

*New additions to the lichen flora of Arunachal Pradesh 

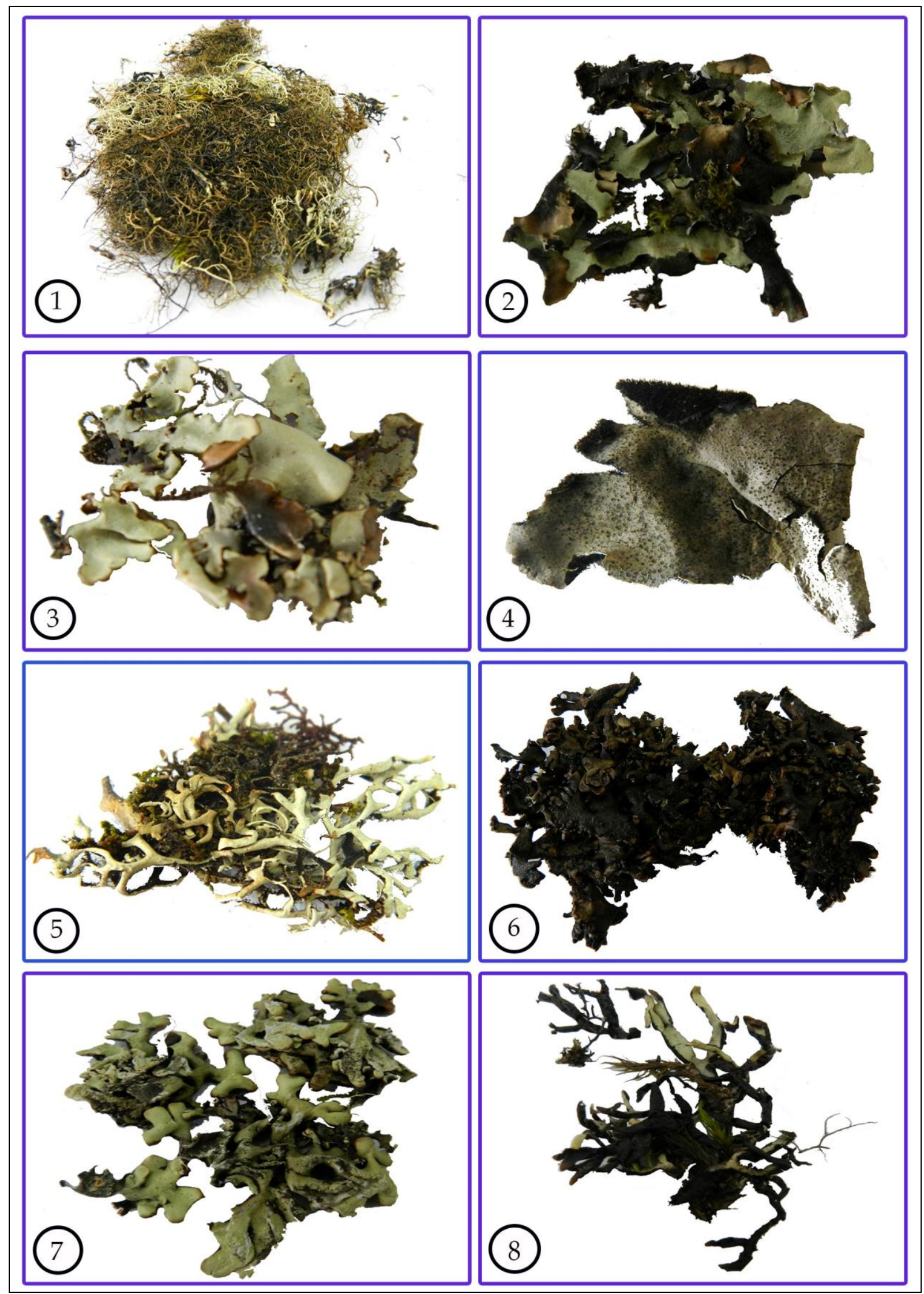

Fig. 2 - New additions of lichens to Arunachal Pradesh. 1 Bryoria tenuis (Dahl) Brodo \& D. Hawksw. 2 Cetrelia collata (Nyl.)W. Culb. \& C. Culb. 3 Cetrelia olivetorum (Nyl.)W. Culb. \& C.Culb. 4 Dermatocarpon vellereum Zschake. 5 Everniastrum neocirrhatum (Hale \& Wirth) Hale. 6 Hypogymnia alpina D.D. Awasthi. 7 Hypogymnia enteromorpha (Ach.) Nyl. 8 Hypogymnia pseudohypotrypa (Asahina) A. Singh. 
Material examined - India, Arunachal Pradesh, West Kameng, Sangti; substratum-tree bark; 23 Sept. 2012; R. Debnath; AUS/JR-RD/APS/12-32, 12-017757(LWG); Tawang, PTTSO; substratum-tree bark; 11 Nov. 2013; R. Debnath; AUS/JR-RD/AP/13-02, LWG Accession no: 29530

Hypogymnia thomsoniana (Mull. Arg.) D.D.Awasthi. (Family: Parmeliaceae)

Fig. 3.9

Thallus to $6 \mathrm{~cm}$ across, branched; lobes to $3 \mathrm{~mm}$ wide; upper side ashy brown to copperbrown, with pycnidia; isidia and soredia absent; Apothecia immature.

Basionym - Parmelia thomsoniana Muller Argoviensis, Flora 74: 379.1891.

Known distribution - Reported from India (Sikkim).

Material examined - India, Arunachal Pradesh, West Kameng, Sangti; substratum- tree bark; 23 Sept. 2012; R. Debnath; AUS/JR-RD/APS/12-25, 12-017790(LWG).

Myelochroa metarevoluta (Asahina) Elix \& Hale. (Family: Parmeliaceae)

Fig. 3.10

Thallus adnate, to $4 \mathrm{~cm}$ across; lobes to $4 \mathrm{~mm}$ wide; upper side whitish grey, postulatesorediate; lower side rhizinate; medulla yellow. Apothecia and pycnidia not known.

Basionym - Parmelia metarevoluta Asahina. J. Jap. Bot. 35: 97.1960. fig.1.

Known distribution - reported from India (Manipur, Meghalaya, Sikkim), China, Japan and North America.

Material examined - India, Arunachal Pradesh, Tawang, adjacent areas of Nagula Lake; substratum-rock; 25 Sept. 2012; R. Debnath; AUS/JR-RD/APNL/12-611(AUS), 12-017772(LWG); Tawang, adjacent areas of Sela lake; substratum-rock; 10 Nov. 2013; R. Debnath; AUS/JRRD/AP/13-79, 15-025663(LWG); LWG Accession no: 32048

Parmelinella chozoubae (Kr. P. Singh \& Sinha) Elix \& Pooprang.(Family: Parmeliaceae) Fig. 3.11

Thallus adnate, to $5 \mathrm{~cm}$ across; lobes sublinear, to $4 \mathrm{~mm}$ wide, truncate, ciliate in axils; upper side grey, lacking isidia and soredia; lower side black. Apothecia laminal; ascospores (9-) 13-17 x

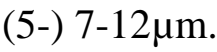

Basionym - Parmelina chozoubae Kr. P. Singh \& Sinha, Nord. J. Bot. 13: 463.1993, p. 464.fig.1: and Singh \& Sinha 1994: 267, p. 256, Pl. 16, fig. 6.

三 Parmelina metarevoluta (Asahina) Hale. Phytologia 28: 483. 1974; Hale 1976d: 36.p. 37. fig. $17 \mathrm{~b}$.

Known distribution - reported from India (Nagaland), Thailand.

Material examined - India, Arunachal Pradesh, West Kameng, Dirang; substratum-tree bark; 22 Sept. 2012; R. Debnath; AUS/JR-RD/APK/12-01, 12-017739(LWG); LWG Accession no: 29514

Parmotrema praesorediosum(Nyl.) Hale. (Family: Parmeliaceae)

Fig. 3.12

Thallus adnate, to $10 \mathrm{~cm}$ across; lobes $5-8 \mathrm{~mm}$ wide, eciliate; upper side grey to darker, emaculate, soralia usually marginal, linear or crescent-shaped; soredia granular; lower side centrally black. Apothecia to $4 \mathrm{~mm}$ in diam., imperforate; ascospores 15-21 x 7-10 $\mu \mathrm{m}$.

Basionym - Parmelia praesorediosa Nylander, Sert. Lich. Trop. Labaun, Singapore: 18. 1891: Hale 1965: 258, Pl. 5, fig. 19: D. Awasthi 1976: 215, Pl. 12, fig. 3.

三Parmelia neglecta Asahina, J. Jap. Bot. 17: 71.1941.

Known distribution - reported from tropical to lowermost temperate regions (Andhra Pradesh, Assam, Himachal Pradesh, Karnataka, Kerala, Madhya Pradesh, Maharashtra, Manipur, Nagaland, Orissa, Rajasthan-Mt Abu, Sikkim, Tamil Nadu- Nilgiri and Palni Hills, Uttaranchal, West Bengal-Jalpaiguri District), Nepal, Sri Lanka, SE Asia, Indonesia and Fiji, Africa. North, Central and South America, Australia, Papua Guinea and New Calendonia.

Material examined - India, Arunachal Pradesh, Tawang, PTTSO; substratum- tree bark; 24 Sept.2012; R. Debnath; AUS/JR-RD/APPT/12-16, 12-017743(LWG) 

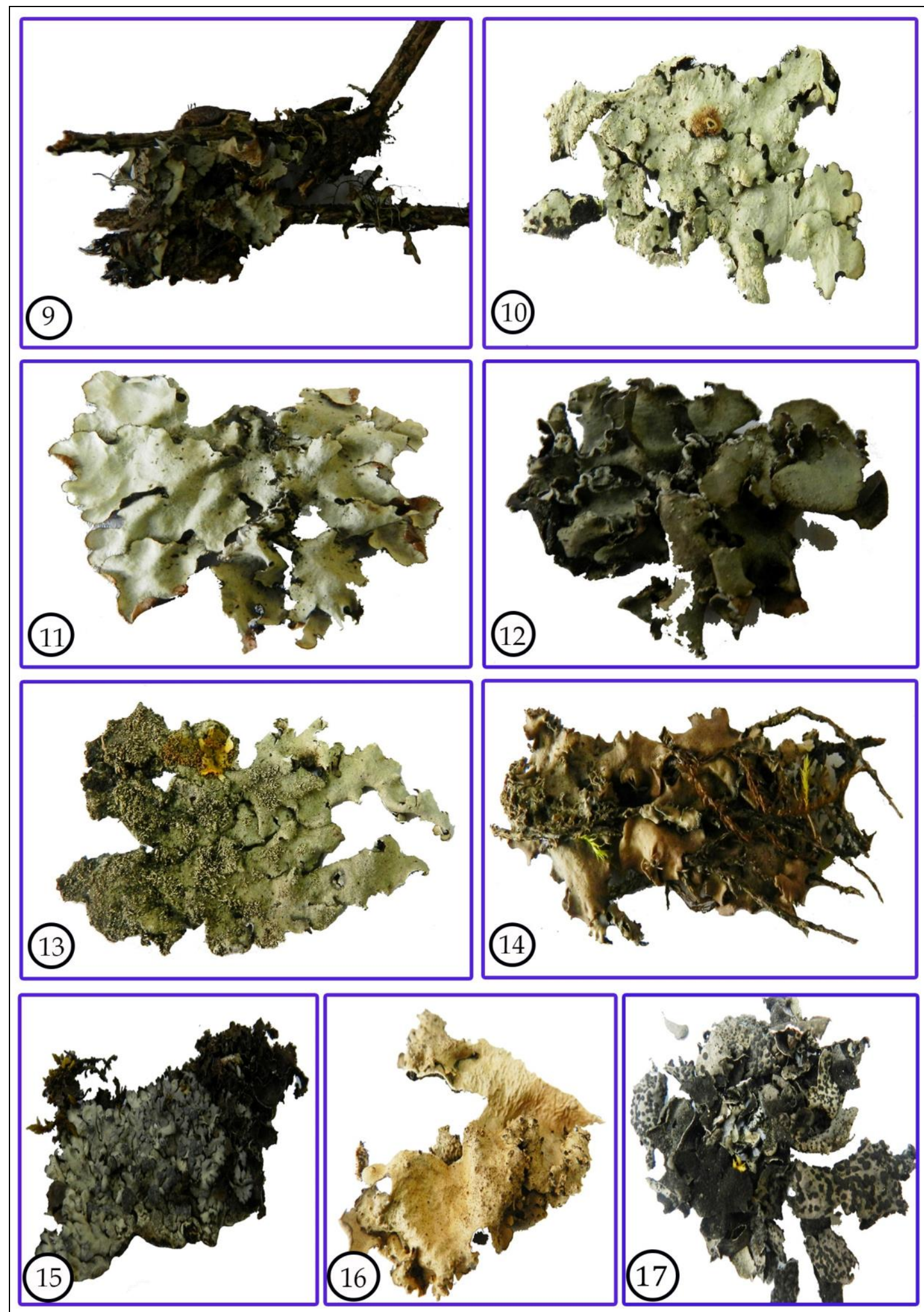

Fig. 3 - New additions of lichens to Arunachal Pradesh. 9 Hypogymnia thamsoniana (Mull. Arg.) D.D. Awasthi. 10 Myelochroa metarevoluta (Asahina) Elix \& Hale. 11 Parmelinella chozoubae (Kr. P. Singh \& Sinha) Elix \& Pooprang. 12 Parmotrema praesorediosum (Nyl.) Hale. 13 Parmotrema saccatilobum (Taylor) Hale. 14 Peltigera collina (Ach.) Schard. 15 Physcia caesia (Hoffm.) Furnr. 16 Sticta nylanderiana Zahlbr.17 Umibilicaria vellea (L.) Ach. Em. Frey. 
Thallus to $10 \mathrm{~cm}$ across; lobes 4-10 $\mathrm{mm}$ wide, involute-tubular (saccate), ecilitate; upper side grey, emaculate, cracked at centre, isidiate; isidia granular to filiform; Indian specimens sterile.

Basionym - Parmelia saccatiloba Taylor, London J. Bot. 6:174.1847; Hale 1965: 262; D. Awasthi 1976: 219, Pl.9, fig 3.

Known distribution - widespread in tropical and subtropical regions of India (Andaman Islands, Assam, Goa, Sikkim, Nagaland, Uttaranchal, West Bengal-Calcutta and Darjeeling District), China, Taiwan, Singapore, Australia, Fiji, Pacific islands.

Material examined - India, Arunachal Pradesh, Tawang, adjacent areas of Nagula lake; substratum-tree bark; 25 Sept. 2102; R. Debnath; AUS/JR-RD/APNL/12-612, 12-017771(LWG); LWG Accession no: 29501

Peltigera collina (Ach.) Schard. (Family: Peltigeraceae)

Fig. 3.14

Thallus adnate, to $3 \mathrm{~cm}$ across; lobes 4-6(-12) $\mathrm{mm}$ wide; upper side yellowish-brown, scabrid, etomentose, marginally soraliate with granular soredia; Indian specimens sterile.

Basionym - Lichen collinus Acharius. Lich. Svec. Prodr.: 162.1798.

$\equiv$ Peltigera scutata (Dicks.) Duby, fide: Thomson Amer, Midl. Nat. 44: 16.1950 .

Known distribution - reported from subtropical to lower temperate regions of India (Sikkim, Tamil Nadu-Nilgiri and Palni Hills, Uttaranchal), China, Central Europe and North America.

Material examined - India, Arunachal Pradesh, West Kameng, Sangti; substratum-rock; 23 Sept. 2012; R. Debnath; AUS/JR-RD/APS/12-40(AUS).

Physcia caesia (Hoffm.) Furnr. (Family: Physciaceae)

Fig. 3.15

Thallus 5-7 $\mathrm{cm}$ across; lobes $3 \mathrm{~mm}$ wide; upper side whitish grey to darker, white-maculate; soralia terminal on short lobes, capitate to lip-shaped. Apothecia to $1.5 \mathrm{~mm}$ in diam.

Basionym - Lichen caesius Hoffmann. Enumeratio Lich.: 65. 1784

$\equiv$ Physcia wainioi Rasanen.Medd. Soc. Fauna Fl. Fenn. 46: 166.1921.

Known distribution-reported from upper temperate regions of India (Jammu \& Kashmir, Manipur, Rajasthan, Uttaranchal), Nepal, Europe and N. America.

Material examined - India, Arunachal Pradesh, Tawang, adjacent areas of Nagula Lake; substratum-rock; 25 Sept. 2012; R. Debnath; AUS/JR-RD/APNL/12-56, 12-017731(LWG); LWG Accession no: 29509

Sticta nylanderiana Zahlbr. (Family: Lobariaceae)

Fig. 3.16

Thallus horizontal, loosely adnate, to $14 \mathrm{~cm}$ across; lobes 3-30 $\mathrm{mm}$ wide; upper side pale grey to darker, lacking isidia and soredia; lower side pale brown to brown, cyphellate: cyphellae initially minute, later 0.4-2 $\mathrm{mm}$ in diam., saucer shaped.

$\equiv$ Sticta damaecornis $*$ S. platyphylla Nylander. Syn. Lich. 1: 357.1860, non Massalongo ex anno 1853. Type: (Nepal. Himalaya) alt. 12000ft. (=3600). Hooker f. \& Thomson 1963(H-NYL 37652-lectotype).

Known distribution - widespread in temperate regions of India (Himachal Pradesh, Manipur, Sikkim, Nagaland, Tamil Nadu and Uttaranchal), Bhutan, Nepal and China.

Material examined - India, Arunachal Pradesh, Tawang, PTTSO; substratum-tree bark; 24 Sept. 2012; R. Debnath; AUS/JR-RD/APPT/12-11, 12-017755(LWG); Tawang, PTTSO; substratum-tree bark; 11 Nov. 2013; R. Debnath; AUS/JR-RD/AP/13-62; LWG Accession no: 29535.

Umbilicaria vellea(L.) Ach. (Family: Umbilicariaceae)

Fig. 3.17

Thallus monophyllous, umbilicate, $2.5-12 \mathrm{~cm}$ across, rigid; margin entire to incised; upper side grey to blackish grey, smooth, areolate, pruinose; lower side black, to brownish black; Apothecia rare, to $3 \mathrm{~mm}$ in diam.

Basionym - Lichen velleus Linnaeus, Sp. Pl.: 1150. 1753. 
Known distribution - reported from temperate regions of India (Jammu \& Kashmir, Himachal Pradesh, Uttaranchal), Africa, Bhutan, Europe, Nepal, Sri Lanka, cosmopolitan in northern arctic regions and New Zealand.

Material examined - India, Arunachal Pradesh, Tawang, adjacent areas of Nagula Lake; substratum-rock; 25 Sept. 2012; R. Debnath; AUS/RD/APNL/12-64, 12-017736(LWG).

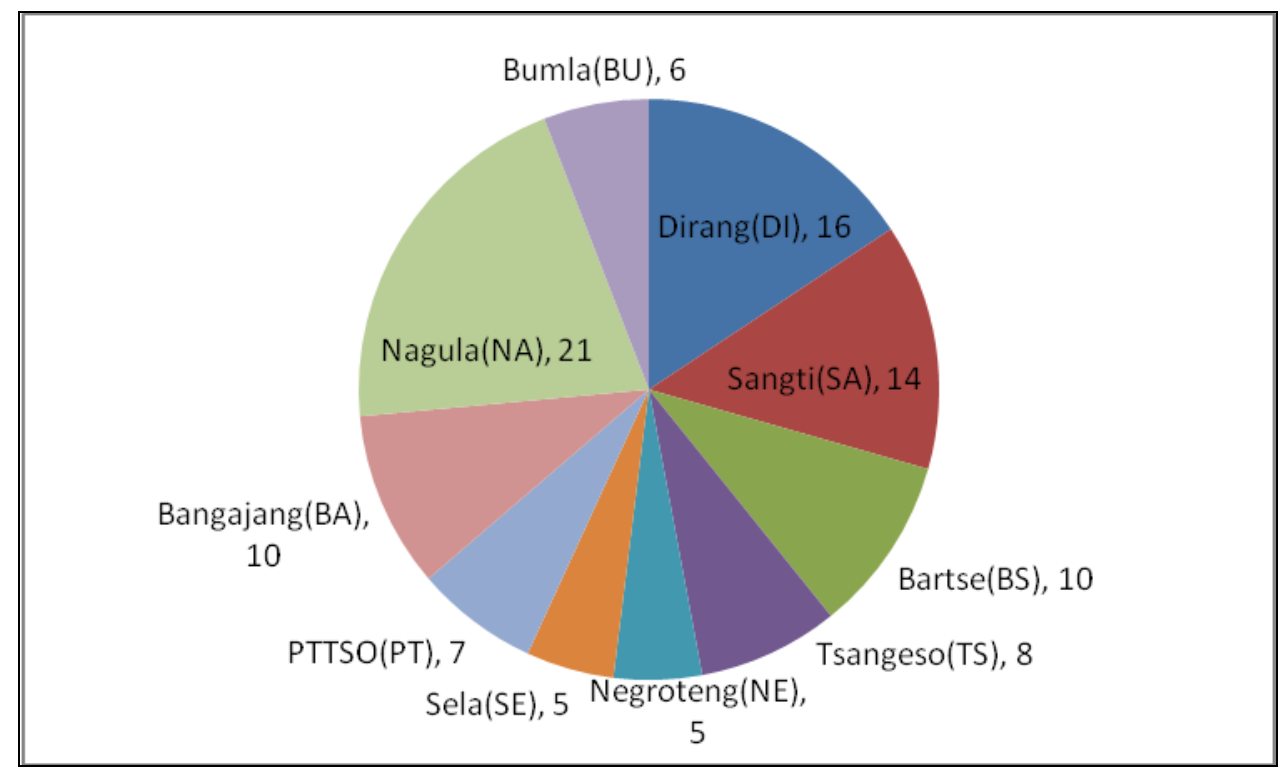

Fig. 4 - Number of species from different study sites

\section{Discussion}

The high elevation, ample sunlight, low temperature and less human disturbances provide suitable environment for the growth of macrolichens. Foliose and fruticose lichens need adequate light condition for their growth. According to James et al. 1977, Broad 1989, Wolseley \& Pryor 1999, the fruticose lichens are most light sensitive. Based on the annotated checklist (Singh \& Sinha 2010), recent reports of Bajpai et al. 2016, Singh et al. 2018, Sinha et al. 2018 the present research led to 17 species belonging to 12 genera and 5 families as new additions to the lichen flora of Arunachal Pradesh.

\section{Note added at proof stage}

While the manuscript was under review, few more works (Bajpai et al 2016, Singh et al 2018, Sinha et al 2018) on the lichen diversity of the state appeared and accordingly the present list of new additions to the lichen flora are based on these reports.

\section{Acknowledgements}

The authors are thankful to the Director of National Botanical Research Institute, Lucknow, for providing laboratory facilities. Thanks are due to Forest department, Arunachal Pradesh and Defence organization for granting necessary permission for the survey and collection. Mr. Sanam is thanked for help with the sample collection. The authors acknowledge Department of Biotechnology, for financial support through DBT-Twinning program (BT/183/NE/TBP/2011, Dtd.23/04/2012). RD thanks UGC, New Delhi for BSR fellowship.

\section{References}

Awasthi DD. 1961 - Some foliose and fruticose lichens from Assam and North-East Frontier Agency of India. Proceedings of the Indian Academy of Science, Sect. 54B (1), 22-44.

Awasthi DD. 1982 - Lichen genus Cetraria in India and Nepal. Bulletin of the Botanical Survey of India 24, 1-27. 
Awasthi DD. 1988 - A key to the macrolichens of India and Nepal. Journal of Hattori Botanical Laboratory 65, 207-302.

Awasthi DD. 1991 - A key to the microlichens of India, Nepal and Sri Lanka. Biblioth. The Lichenologist 40, 1- 337.

Awasthi DD. 2007 - A Compendium of the Macrolichens from India, Nepal and Sri Lanka. Bishen Singh Mahendra Pal Singh, Dehradun, India.

Bajpai R, Shukla V, Singh CP, Tripathi OP et al. 2016-Lichen Community Composition in Tawang District of Arunachal Pradesh, Tool for Long-Term Climate Change Monitoring. Proceedings of the National Academy of Sciences, India. Section B: Biological Sciences. Doi 10.1007/s40011-016-0830-z

Broad K. 1989 - Lichen in southern woodland. Forest Officer, Forestry Commission, Handbook 4, London: Her Majestry's stationery office.

Culberson CF.1972 - Improved conditions and new data for the identification of lichen products by a standardized thin - layer chromatographic method. Journal of Chromatography 72, 113125.

Culberson CF, Kristinsson H. 1970 - A standardized method for the identification of lichen products. Journal of Chromatography 46, 85-93.

Daimari R, Hazarika N, Hoque RR, Nayaka S, Upreti DK. 2014 - New records of epiphytic lichens from three districts of Assam, India. Indian Forester 140(8), 807-811.

Dey AK, Mishra GK, Rout J, Upreti DK. 2015 - An enumeration of epiphytic lichens from Hojai sub -divison of Nagaon district, Assam, India. International Journal of Advance Research in Biological Sciences 2(10), 111-115.

Devkota S, Chaudhary RP, Werth S, Scheidegger C. 2017 - Indigenous knowledge and use of lichens by the lichenophilic communities of the Nepal Himalaya. Journal of Ethnobiology and Ethnomedicine 13, 15.

Devi RKS, Rout J, Upreti DK, Nayaka S, Pinokiyo A. 2015 - New records of lichens from Manipur State, North-East India. Mycosphere 6(6), 796-813.

Divakar PK, Upreti DK. 2005 - Parmeloid lichens in India. Bishen Singh and Mahendra Pal Singh, Dehradun, India, pp. 1-448.

Dubey U, Upreti DK, Rout J. 2007 - Lichen flora of along town, West Siang district, Arunachal Pradesh. Phytotaxonomy 7, 21-26.

Dubey U. 2009 - Assessment of lichen Diversity and Distribution for Prospecting the Ecological and Economic potential of lichens in and around Along town, West Siang district, Arunachal Pradesh. Department of Ecology and Environmental Science, School of Environmental Science, Assam University, Silchar.

Ingle KK, Trivedi S, Nayaka S, Upreti DK. 2017 - The lichen genera Dictyomeridium and Polymeridium (Trypetheliales: Trypetheliaceae) in India. Taiwania 62(1), 50-54.

James PW, Hawksworth DL, Rose F. 1977 - Lichen communities in the British Isles: a preliminary conspectus in Seaward, M.R.D.(eds). Lichen Ecology, London Academic Press, pp. 295-413.

Logesh A, Chinlampianga M, Shukla A, Upreti DK. 2017 - Studies on Lichens of Mizoram, Northest India. Proceedings of the National Academy of Sciences, India. Section B: Biological Sciences 87(2), 445-457.

Kaul RN \& Haridashan K. 1987 - Forest types of Arunachal Pradesh-a preliminary study. Journal of Economic and Taxonomic Botany 9, 379-389.

Panda M, Murthy TVR, Samal RN, Lele N, Patnaik AK, Mohan PK. 2017 - A comparative study of mangnicolous lichens their distribution inside Bhitarkanika National Park (Odisha), India. Studies in Fungi 2(1), 1-13.

Pinokiyo A, Singh KP, Singh JS. 2008 - Diversity and distribution of lichens in relation to altitude within a protected biodiversity hot spot, North-East India. The Lichenologist 40(1), 47-62.

Rai H, Khare R, Gupta RK, Upreti DK. 2011 - Terricolous lichens as indicator of anthropogenic disturbances in a high altitude grassland in Garhwal (Western Himalaya), India. Botanica Orientalis - Journal of Plant Science 8, 16-23. 
Rout J, Kar A, Upreti DK. 2004a - Lichens of Sessa Orchid Sanctuary, West Kameng, Arunachal Pradesh. Phytotaxonomy 4, 38-40.

Rout J, Kar A, and Upreti, DK. 2004b - Traditional remedy for kidney stones from a high altitude lichen: Cladonia rangifera (L.) Wigg. (Reindeer moss) of Eastern Himalaya. Ethnobotany 17, 164-166.

Rout J, Rongmei R, Upreti, DK. 2005 - Epiphytic lichen flora of a pristine habitat (NIT campus) in Southern Assam, India. Phytotaxonomy 5, 117-119.

Rout J. 2007 - Air pollution biomonitoring: Lichens as an indicator species. In: Biodiversity and environmental Biotechnology. Scientific publishers, Jodhpur, 563.

Rout J, Das P, Upreti DK. 2010 - Epiphytic lichen diversity in a Reserve Forest in southern Assam, northeast India. Tropical Ecology 51, 281-288.

Rout J, Devi SRK, Upreti DK, Pinokiyo A. 2013 - A preliminary observation on the lichens of Keirao Wangkhem, Imphal East, Manipur, India. In: Plant Biocon (eds.) Proceedings on Plant Diversity Resources of Indo- Burma Biodiversity Hotspot and their Conservation, 215-220.

Santesson J. 1973 - Identification and isolation of lichen substances. In: Ahmadjian, V, Hale ME (eds), The lichens. Academic press, London, pp. 633-652.

Singh KP, Pinokiyo A. 2004 - Four foliicolous lichens new to Indian flora. Geophytology 33(1-2), 119-121.

Singh KP, Sigh P, Sinha GP. 2018 - Lichen diversity in the Eastern Himalaya biodiversity hotspot region, India. Cryptogam Biodiversity and Assessment 71-114.

Singh KP, Sinha GP. 2010 - Indian Lichens: An annotated Checklist. Botanical Survey of India, Ministry of Environment and Forest, Salt Lake City, Kolkata.

Singh KP, Sinha GP. 1997 - Lichens. In: Floristic Diversity and Conservation Strategies in India, Vol. 1 Cryptograms and Gymnosperms (V. Mudgal and P.K. Hajra, eds.). Botanical Survey of India, New Delhi, India, pp. 195-234.

Sinha GP, Jagadeesh Ram TAM.2011 - Lichen diversity in Sikkim. In: Arrawatia M.L. \& Tambe S (Editors)-Biodiversity of Sikkim Exploring and Conserving a Global Hotspot. Department of Information and Public Relations, Government of Sikkim, Gangtok, pp. 13-29.

Sinha GP, Nayaka S, Joseph S. 2018 - Additions to the checklist of Indian lichens after 2010. Cryptogam Biodiversity and Assessment 197-206.

Upreti DK. 1985a - Studies on the lichen genus Baeomyces from India. Geophytology 15(2), 159163.

Upreti DK. 1985b - The lichen genus Cladia from India and Nepal. Journal of Economic and Taxonomic Botany 7(3), 722-724.

Upreti DK. 1997 - Diversity of the Himalayan Lichens. In: S.C. Sati, J. Saxena and R.C. Dubey (Eds.). Himalayan Microbial Diversity (Part 2). Recent Researches in Ecology, Environment and Pollution. Today and Tomorrow's Printers \& Publishers, New Delhi, pp. 339-347.

Upreti DK. 1998 - Diversity of lichens in India. In: Perspectives in Environment (S.K. Agarwal, J.P. Kaushik, K.K. Kaul and A.K. Jain, eds.). APH Publishing Corporation, New Delhi, India, pp. 71-79.

Upreti DK, Debnath R, Uppadhyay V, Rout J. 2015 - Diversity and distribution of lichens in north and west districts of Tripura. Phytotaxonomy 14, 122-129.

Wolseley PA, Moncrieff C, Aguirre HB. 1994 - Lichens as indicators of environmental stability and change in the tropical forests of Thailand. Global Ecology and Biogeography Letters 4, 116-123.

Wolseley PA, Pryor KV. 1999 - The lichens of epiphytic twig communities on Quercus petrarea in a welsh Woodland site (Tycanal) fir evaluating environmental changes. Lichenologist 31(1), $41-61$. 